\title{
"First Week Is Editorial, Second Week Is Algorithmic": Platform Gatekeepers and the Platformization of Music Curation
}

Social Media + Society

October-December 2019: |-I1

(C) The Author(s) 2019

Article reuse guidelines: sagepub.com/journals-permissions DOI: 10.1 I77/2056305।19880006 journals.sagepub.com/home/sms (S)AGE

\author{
Tiziano Bonini' ${ }^{1}$ and Alessandro Gandini ${ }^{2}$
}

\begin{abstract}
This article investigates the logics that underpin music curation, and particularly the work of music curators, working at digital music streaming platforms. Based on ethnographic research that combines participant observation and a set of interviews with key informants, the article questions the relationship between algorithmic and human curation and the specific workings of music curation as a form of platform gatekeeping. We argue that music streaming platforms in combining proprietary algorithms and human curators constitute the "new gatekeepers" in an industry previously dominated by human intermediaries such as radio programmers, journalists, and other experts. The article suggests understanding this gatekeeping activity as a form of "algo-torial power" that has the ability to set the "listening agendas" of global music consumers. While the power of traditional gatekeepers was mainly of an editorial nature, albeit data had some relevance in orienting their choices, the power of platform gatekepeers is an editorial power "augmented" and enhanced by algorithms and big data. Platform gatekeepers have more data, more tools to manage and to make sense of these data, and thus more power than their predecessors. Platformization of music curation then consists of a data-intense gatekeeping activity, based on different mixes of algo-torial logics, that produces new regimes of visibility. This makes the platform capitalistic model potentially more efficient than industrial capitalism in transforming audience attention into data and data into commodities.
\end{abstract}

\section{Keywords}

music streaming platforms, ethnography, music curation, algo-torial power, platform gatekeeping

\section{Introduction}

This article investigates the logics that underpin music curation, and particularly the work of music curators, at music streaming platforms. In the midst of the platformization of cultural production and consumption (Nieborg \& Poell, 2018), music occupies a key positioning.

Following the disruption engendered by $\mathrm{P} 2 \mathrm{P}$ technologies (e.g., Napster) in the early 2000s, which led to a dematerialization of music as a commodity and marked the beginning of an era of wide availability of music content, we are now witnessing a re-intermediation of music consumption practices controlled by commercial music streaming platforms. These platforms allow consumers to access a large database of content regulated by top-down assessments of bottom-up user practices. Within this context, music curation has been the object of scarce academic attention, mostly due to the difficulties in accessing the research field (Seaver, 2017), and despite the important role curation plays in the making of music taste (Eriksson, Fleisher, Johansson, Snickars, \&
Vonderau, 2019; Fleischer \& Snickars, 2017). Researchers have studied the role played by algorithms in music curation (Barna, 2017; Morris, 2015) and in the cultural industries more in general (Napoli, 2014). However, the specific intermingling of human and algorithmic processes in music curation remains relatively underexplored.

To pursue this investigation, we take inspiration from the work of Gans (1979) and others in the study of "gatekeeping" at newspapers and television newsrooms, extending this approach to the study of music streaming platforms. We question the extent to which music curation processes in the present-day music industry are similar to the kind of gatekeeping that characterizes established forms of media

\footnotetext{
'University of Siena, Italy

${ }^{2}$ University of Milan, Italy

Corresponding Author:

Tiziano Bonini, Department of Social, Political and Cognitive Sciences, University of Siena, Via Roma 56, Siena 53100, Italy.

Email: tiziano.bonini@unisi.it
} 
production and examine the meeting between human and algorithmic logics. Based on ethnographic research that combines participant observation and a set of interviews with key informants, the article demonstrates that digital music curation consists of partly editorial, partly algorithmic logics whereby human agency blends with the automated functioning of algorithmic infrastructures in ways that exert new forms of power based on this intermingling. Thus, we argue that music streaming platforms, in their combination of proprietary, algorithmically driven, and human curation, represent the "new gatekeepers" of an industry previously dominated by human intermediaries such as radio programmers, journalists, and other experts. We suggest that this gatekeeping activity is a form of "algo-torial power" that has the ability to set the "listening agendas" of global music consumers. Corroborating Bucher's (2017) analysis, the article shows the advantages of moving beyond algorithms as "black boxes" to study the social and cultural practices that underpin emergent algorithmic infrastructures. Exploring how algorithmic and human curation work at music streaming platforms intermingle and blend is a key step in the quest to understand how contemporary music curation is being platformized.

\section{The Great Shift: From Human to “Platform" Gatekeepers}

The launch of the file-sharing service Napster in 1999 marked a "great shift" in music consumption and curation: from an environment dominated by traditional gatekeepers-music journalists, radio programmers, and other experts - to a disintermediated environment. Listeners were suddenly offered the possibility to seamlessly exchange files with others and access a virtually infinite, largely illegal database of digital music (Hesmondhalgh \& Meier, 2018). For a few years, like pirate and free radio stations in the 1960s-1980s, the Internet was truly capable of disintermediating music consumption: people were consuming and exchanging music outside the "fences" of commercial audience rating circuits. This not only affected the music industry, but quickly expanded to all traditional cultural industries: the ability of broadcasting institutions to successfully situate audiences (and render their desires predictable) had declined. As Arvidsson and Bonini (2015, p. 2) maintain, "an audience commodity became valuable precisely by being situated 'in front of the radio' so to say, so that it could be relied on to reproduce a particular consumption norm with calculable predictability." For a while, the diffusion of independent web radios, blogs, and $\mathrm{p} 2 \mathrm{p}$ music consumption shielded music listeners from audience quantification and commodification. But, the subsequent rise of music streaming platforms such as Spotify, Apple Music, and others reversed this shift and started a process of re-intermediation of music consumption practices, re-locating music audiences into newly fenced digital environments. In 2008, file sharing (including music) accounted for one-third of US Internet traffic. By 2014, it had already fallen to $8 \%$ (Fiegerman, 2014). As p2p music sharing declined, platforms such as Apple and Spotify increased their subscriber base. In 2018, these platforms respectively had 50 and 97 million paid subscribers. As Gillespie (2018) notes, "to be free of intermediaries, we accepted new intermediaries" (p. 16). We hereby consider these "new intermediaries" as the infrastructures that informational capitalism has built to (re)capture attention and extract value. In this context, music streaming platforms are a native infrastructure of the digital music industry, just like commercial broadcasting used to be a native infrastructure of the 20th-century music industry.

An article in the Financial Times has described the arrival of music streaming platforms as a "sea change" (Shah, 2017). In this context, a particular platform feature stands out: playlists. As noted by Prey, playlists "are a repackaging of music in a form native to streaming platforms" (Prey, 2018). Streaming playlists are used by nearly $60 \%$ of US music streamers, according to Nielsen Music. Top 40 commercial radio programmers today usually play what's popping on Spotify and Apple Music, instead of breaking new songs themselves (Shah, 2017). Daniel Ek, the founder of Spotify, claimed that "over $30 \%$ of consumption on Spotify is now a direct result of recommendations made by the platform's own algorithms and curation teams," something that, he said, "puts Spotify in control of the demand curve" (Ingham, 2018b). Hogan (2015), based on data from research conducted on 1,500 British, French, and US music listeners, claimed that music consumption on streaming music platforms is shifting from albums to playlists. Out of the total sample, $45 \%$ said they listen mainly to playlists and $21 \%$ said they listen mainly to albums. As for subscribers to music streaming services, $68 \%$ said they mainly listen to the playlists suggested by platforms. ${ }^{1}$

Spotify and Apple Music both offer large databases of songs, but what sets them apart from each other is their different selection and curation of playlists. Eriksson et al. (2019) recount that between 2013 and 2015, Spotify began to transform itself "from being a simple distributor of music to the producer of a unique service" (p. 61). It may be argued, thus, that curation represents the distinctive service (the commodity) that music streaming platforms offer to their user-base (Fleischer, 2017).

As a result, a new class of powerful gatekeepers is emerging, which give meaning and value to certain music tracks and artists and mediate tastes, moods, and lifestyles, converting them into valuable objects of consumption in the form of playlists. However, research on music curators, their work and their powerful role in the industry, is still in its infancy. On one hand, current research focuses on the macrosocial changes that the music industry experienced as a result of the advent of platforms (Hesmondhalgh \& Meier, 2018; Mulligan, 2015). On the other hand, significant attention is paid to the role of algorithms, particularly recommendation 
systems (Barna, 2017; Hallinan \& Striphas, 2016), which Morris (2015) describes as "infomediaries" that intervene at the intersection of data mining, taste curation, and audience manufacturing. Yet, as underlined by Airoldi, Beraldo, and Gandini (2016), it is necessary to understand the role of algorithmic logics, particularly with regard to digital music consumption - not in isolation, but through their interplay with social logics and human interventions.

The study of the relationship between algorithmic affordances and human agency has been object of much less attention in current scholarship, as algorithms have often been considered as inaccessible infrastructures of code, or "black boxes" (Pasquale, 2015), away from public scrutiny. On the contrary, following Seaver $(2013 ; 2018)$ and Bucher (2016), we argue that it is not only necessary to expand our knowledge and understanding of algorithms and the outcomes they generate but also to investigate the social and cultural constructs that lie behind them. We must look, as Beer (2017) puts it, "inside the algorithmic workings of the 'black box society' (Pasquale, 2015)" (p. 10) - and, following Kitchin (2017), we contend there is a need to "unpack the full socio-technical assemblage of algorithms" (2017, p. 25), without overstating the relevance of technology alone.

To this end, we turn to music streaming platforms as a particularly interesting example to study the processes of curation and selection that underpin the platformization of cultural artifacts. Selection, together with datafication and commodification, is recognized by van Dijck, Poell, and de Waal (2018, p. 40) as one of the three key mechanisms of platformization. We argue that investigating how music selection and curation work on music streaming platforms allows us to make a first, thin, crack in the black box of platforms. It enhances our understanding of the kind of power these platforms are exerting on the consumers and industries in which they intervene. The notion of "gatekeeping," we contend, represents a useful interpretative frame for this inquiry.

\section{Music Curators as Gatekeepers?}

The study of gatekeepers has a long tradition in media and communication studies (Lewin, 1947; White, 1950). The technological, cultural, and social filters that determine the editorial choices made in the newsrooms of newspapers and television channels have been extensively investigated in media research (Altheide, 1976; Gans, 1979; Gitlin, 1980; Schlesinger, 1978; Tuchman, 1978). The concept of gatekeeping was originally used to describe the news selection process that operates under several layers of influence. According to Shoemaker, Vos, and Reese (2008), gatekeeping is "the process of selecting, writing, editing, positioning, scheduling, repeating and otherwise massaging information to become news" (p. 73). Since its adoption in media theory (Lewin, 1947; White, 1950), this concept has extended to the media industries more broadly, to include all those key figures that influence the processes of production and distribution of cultural artifacts.

Gatekeeping is nevertheless a problematic concept that has been extensively critiqued. Here, we acknowledge its limitations, already underlined by McQuail (1994), among others. With the spread of the Internet and, later, of social media, many have attributed gatekeeping capabilities to audience members and networked publics alike. Meraz and Papacharissi (2013), for instance, found strong evidences for "networked gatekeepers" among non-elite digital activists.

Some also investigated the role of gatekeeping activities in the context of digital networks, theorizing the attributes of "network gatekeeping" (Barzilai-Nahon, 2008), while others described algorithms as "gatekeepers" and compared the traditional gatekeeping practices that happened in the journalistic newsrooms with the filtering role played by algorithms on Facebook and Twitter. Tufekci (2015, p. 209), for example, claimed that

Algorithms, or computational processes that are used to make decisions, are often deployed as gatekeepers; in this function, they are somewhat similar to the role of a newspaper editor, but possess important differences from their offline, non-interactive and non-computational counterparts. Hence, algorithmic gatekeeping raises significant yet novel issues in many realms.

However, the specific workings of this "algorithmic gatekeeping" remain to be investigated in depth. Here, we decided to adopt the notion of gatekeeping to describe the activities of music curators working at streaming platforms, for two reasons. First, it has emerged "from below," so to speak, from the interviews we held with our informants. Many defined themselves as "gatekeepers" or described the selection activities of human and non-human music curators as forms of "gatekeeping." Second, the rising popularity of commercial music streaming platforms puts a new emphasis on the power of the "gates" that filter and shape the circulation of digital music. We call these "platform gatekeepers."

\section{Platform Gatekeepers: A Profile}

We call "platform gatekeepers" all those workers within music streaming platforms, who are able to decide, filter, and select what to expose listeners to and which songs to direct their attention to. We focus in particular on the human music curator: this role did not exist at music streaming platforms before 2014-2015. Eriksson et al. (2019, p. 61) situate the curatorial turn after Spotify acquired Tunigo and Echo Nest, in 2014, 8 years after the company was founded. Google Play Music also began employing human curators in late 2014, 3 years after its birth. Apple Music hired the first human curators in 2015 (Ugwu, 2016). According to Shah (2017), Spotify employs the highest number of curators (approximately 150, up from 50 as reported by Ugwu in 2016). According to Ugwu (2016), Google Play has 20 
full-time curators plus additional freelancers, while Apple Music has "more than 12," plus additional freelancers. In 2016, Deezer revealed to The Guardian that they employed 50 editors (Dredge, 2016). These numbers are not completely up to date, and data about other services, such as Tidal and Amazon Music, are not available. However, it seems fair to estimate that there are currently hundreds of these editors working globally, mostly distributed between New York (Google Play Music, Spotify, Tidal, and Amazon Music), Los Angeles (Apple Music), and London (Spotify, Deezer, Google Play Music, and Apple Music). This might be seen as a "global elite" of music specialists that has accrued a large share of power at these companies, insofar as they oversee and ultimately decide on the inclusion and exclusion of music tracks and artists on successful playlists.

Music curators are divided into senior and junior music curators. Seniors are also responsible for the company's content strategy for a specific music genre (e.g., "global head of Latin music") and work on the creation and management of the most popular playlists. Each curator is an expert in a specific genre or sub-genre; their daily job mostly consists of assembling different songs into playlists, and they usually belong to a specific curatorial team with whom they discuss editorial choices. One of our informants $(\mathrm{N})$, working at Google Play, told us that

On average, I created about thirty new playlists per month. The new ones were created with the aim to complete the offer of playlists in terms of music genre, historical ages, mood, events or specific festivities and holidays. Every week we also used to update about fifty playlists.

As a cohort, they are mostly university-educated, quite diverse in terms of gender, and despite their geographic dispersion, largely coherent in their account of the practices and cultures of their work.

Most curators, as Shah (2017) showed, have previous work experience within the music industry. Those publicly known among them include former music journalists, radio deejays (such as Sara Sesardic, an editor at Spotify UK, who worked at BBC Radio 2), radio music programmers, former music executives, and managers. Some have previous experience as journalists for online music magazines or were amateur musicians, such as Athena Koumis (Spotify editor of the "Fresh Finds" playlist; Shah, 2017). Others were at once music journalists and musicians, such as Sam Lee, a curator at Deezer, (Dredge, 2016). This was confirmed by our informants. Our informant at Apple Music told us that they have "a very large staff of humans" (sic!) "that come from backgrounds in the industry, essentially, radio or working with labels" (Informant P). Their role is a powerful one, since their decisions influence the fate of artists and music tracks; yet, in turn, they are also influenced by the industry and the specialized music press, as they refine their musical taste by reading music blogs and critic reviews and attending music gigs (Tiffany, 2017), thus keeping themselves constantly updated about insider information, new releases, and trends. In the words of one of our informants, "You know the people, you go to dinner and have drinks with them, you are emailing them stuff every day, you are at the back and forth with them. It's a relationship" (Informant B, digital music promoter). A whole "cottage industry" of pitching companies has popped up, such as Playlist Pump, which claims to assist independent artists with doing "what only major record labels were able to do in the past - offer massive exposure for artists through direct relationships with curators of many of the major playlists featured on Spotify" (Lucerne, 2017).

\section{Research Design: The Field as a "Black Box"}

Our research aimed at investigating the logics of curation at music streaming platforms. To do so, we undertook what may be described as a multi-sited ethnography (Marcus, 1995) constituted by a set of interviews with key informants in the music industry and a short participant observation inside the music department of two public service radio stations, BBC Radio 6 and Rai Radio 2, in London and Rome, in November 2017. We conducted 17 semi-structured interviews ${ }^{2}$ with key informants working in the European music industry in London (United Kingdom), Gothenburg (Sweden), New York (United States), Berlin (Germany), Rome and Milan (Italy), between October 2017 and April 2018. The interviewees are data scientists (2), radio music programmers for public service and commercial broadcasters (3), marketing managers (2), software developer (1), music startup co-founders (2), head of streaming strategies (1), music curators for streaming platforms (2), major and indie music label companies (3), and music manager (1). Among them, some work for platforms such as Apple Music, Spotify, Google Play Music, Tim Music, and Shazam, while others work for record labels such as Sony and Universal or for digital music startups. ${ }^{3}$ The interviews were supplemented with an analysis of "grey literature" on music streaming platforms published in international newspapers, music magazines, and newsletters in recent years, such as The Guardian (Dredge, 2016), The Wall Street Journal (Allen, 2017; Shah, 2017; Ugwu, 2016), and The Verge (Popper, 2015; Tiffany, 2017).

Inspired by Grounded Theory (Glaser \& Strauss, 1967), we produced an array of fieldnotes, interview transcripts, and documents that were analyzed through an iterative process of sense-making (Schwartz-Shea \& Yanow, 2013). Concept development took place during fieldwork, not before it. As Georgina Born once argued, "at the start of the fieldwork you must begin with an open mind" (Szczepanik, 2013, p. 103). Reviewing the data, the intermingling between algorithmic affordances and human agency in music curation became more and more apparent and emerged as our main topic of investigation. 
Yet, trying to access and map the field proved to be challenging. As various researchers have already noted, gaining insight in platforms' internal workings is particularly difficult (Fleischer \& Snickars, 2017; Seaver, 2017). Our initial idea was to visit the headquarters of the most important music streaming companies (Apple, Spotify, Google, Amazon, and Deezer) for a period of participant observation and to interview those who work on the daily production of playlists and the maintenance of algorithms. In practice, however, access to these companies through a formal request was almost always denied. Concerning Spotify, for instance, after 3 weeks of requests and many unanswered emails, a spokesperson told us, "I have to inform you that unfortunately at the moment it is not possible to organize an interview with the editorial team." Similarly, after receiving an enthusiastic reply from Deezer to our initial inquiry, we never received an answer to follow-up emails to schedule a meeting. Others did not reply at all. Thus, we decided to search for key informants who work (or have worked) with or within these companies. This also proved to be difficult, despite the promise of anonymity and confidentiality. In many cases, interviewees were recruited through trusted "brokers" who facilitated a connection between them and us; some ultimately agreed to participate in our research only because contact with us was established through personal connections. As Hannerz (2003) already noted, access to the field is more and more dependent on the entanglement between researchers and "the people in our fields" (p. 58).

In various cases, interviews were preceded by negotiations over what we could, and could not, discuss. In one case, we had to exchange 16 emails to finally convince a software developer from a major digital streaming platform to commit to an interview. One of the informants did accept to be interviewed, via email, but half of his answers were "Can't disclose this piece of information :)."

Alongside these interviews, to substantiate our empirical base with observational data on the kind of power that music streaming platforms exert on other actors in the industry, we did a short participant observation inside the music department of two public service radio stations, BBC Radio 6 and Rai Radio 2, in London and Rome, in November 2017. This consisted of shadowing two music programmers for 3 days in London and for a week in Rome and having both informal and recorded conversations with them and their editorial teams. As a result, we generated an eclectic array of data, consisting of interviews; participant observations; and also music industry news readings, informal talks, and continuous surveying of music curators' Twitter profiles. Our research can be considered as a multi-sited ethnography (Marcus, 1995), in the sense intended by Hannerz (2003):

Interacting with informants across a number of dispersed sites, but also doing field work by telephone and email, collecting data eclectically in many different ways from a disparate array of sources, attending carefully to popular culture, and reading newspapers and official documents. (p. 212)

We thus discovered that it is the whole field of music curation, constituted by the network of digital music companies, that represents the "real" black box, not the proprietary algorithms in isolation.

\section{"First Week Is Editorial, Second Week Is Algorithmic": The Algo-torial Logic of Platform Gatekeeping}

During our work "in the field," the intermingling between algorithmic affordances and human agency in music curation emerged as our main topic of investigation. In this section, we will try to make sense of what we have learnt about it.

The editorial and algorithmic logics are usually conceived as two separated concepts and are clearly recognized by our informants as stand-alone analytical concepts, as already described by Gillespie (2014):

We might consider the algorithmic as posed against, and perhaps supplanting, the editorial as a competing logic. The editorial logic depends on the subjective choices of experts, themselves made and authorized through institutional processes of training and certification or validated by the public through the mechanisms of the market. The algorithmic logic, by contrast, depends on the proceduralized choices of a machine, designed by human operators to automate some proxy of human judgment or unearth patterns across collected social traces. (p. 192)

These two logics, however, are never completely separated in reality: there is always friction between the two. They are difficult to disentangle in the everyday practice of digital music platforms: algorithmic and editorial logics are "stacked" together in interesting ways.

The activity of compiling playlists, either for a radio or a music streaming platform, is, at the same time, both strongly editorially and algorithmically driven. In radio, playlists are generated with the support of Selector and other similar software, while on music streaming platforms, each company has developed its own proprietary software for data analysis. As described in a BuzzFeed article on Google Play Music,

The data is compiled in a Google spreadsheet, with each song in the playlist ranked by "Song Score," a multipoint metric that, like Spotify's PUMA, accounts for things like average play length, skips, and number of thumbs-up or thumbs-down. Editors typically access this data via a Google-designed content management system called Jamza, which, among other things, can recommend songs to add to a playlist based on ones that have already been chosen, or by doing a keyword search. (Ugwu, 2016)

Just as journalists increasingly rely on data analytics suites like Chartbeat, Homegrown, and Parse.ly for their 
gatekeeping activities (Petre, 2015), music streaming platform gatekeepers are also supported by proprietary data analytics tools. For instance, Playlist Usage Monitoring and Analysis (PUMA) breaks down each song on a playlist by things like number of plays, number of skips, and number of saves. PUMA also tracks "the overall performance of the playlist as a whole, with colorful charts and graphs illustrating listeners' age range, gender, geographical region, time of day, subscription tier, and more" (Pelly, 2017).

Yet, editorial decisions still matter significantly: when we asked one of our informants if he could roughly assess how personal taste, editorial choices, and algorithmic suggestions affected his curatorial work, he answered that his choices were " $10 \%$ personal taste-driven, $40 \%$ editoriallydriven, $50 \%$ algorithmically-driven" (Informant N). The weight of one's personal gut in guiding the choices of music programmers has not disappeared, but it has been greatly reduced, in favor of editorial pressure and assistance provided by software.

Similar practices exist at Apple: "From my side, we have algorithms that are producing insights that we've provided to the editorial team as a service" (Informant P, Apple). At Spotify, proprietary playlists can be completely generated by humans, or be totally automated. In fact, as one of Spotify's content editors, Austin Daboh, disclosed, "we have three different types of playlists on Spotify ... we have $100 \%$ handcrafted curated playlists . . algotorial playlists ... then we've got $100 \%$ fully algorithm-based playlists" (Ramirez, 2017).

According to Daboh, a " $100 \%$ algorithmically-generated playlist" is a playlist like "Release Radar" or "Discover Weekly," which are personalized lists of songs generated by algorithms without curatorial intervention. " $100 \%$ handcrafted playlists" are those lists like Rap Caviar that rely on the experience, gut, and knowledge of the top music curators at Spotify. But this distinction is at least ingenuous, because every playlist, whether it is " $100 \%$ handcrafted" or " $100 \%$ algorithm based," contains both logics in an inextricable way: every playlist is algo-torial, much more than the curators themselves believe.

Both playlists like "Rap Caviar" or the mood/situation/ genre-based playlists are edited by humans but they are also strongly supported by data, as are the charts' playlists, while personalized playlists such as Daily Mix, Release Radar, and Discover Weekly are generated by algorithms but are constantly monitored by curators and software developers who manage and improve them. In particular, Discover Weekly is the product of various factors that also indirectly incorporate editorial logics. It is based on collaborative filtering and music structure analysis and also on a system of natural language processing algorithms that crawl through hundreds of music blogs, reviews, and web pages. This means that the selection automatically operated by the algorithms of Discover Weekly is also influenced/shaped by the hundreds of bloggers (amateur and pro/am gatekeepers) and music critics (traditional gatekeepers) scanned by the algorithm (Popper, 2015; Prey, 2017). In other words, Discover Weekly is only partly the product of an algorithmically mediated process, because it also incorporates the editorial choices of the most influential music journalists and bloggers of the music industry. It may be said that Discover Weekly has "subsumed" the social influence of traditional and amateur gatekeepers into its code.

Curation on music streaming platforms in other words is the intermingling process that results from combining human activity "augmented" by algorithms and non-human activity designed, monitored, and edited by humans. Machines (algorithms) do not replace nor are they separated from the work of human curators. Spotify has continued to hire music curators while investing in "technology for music intelligence" (Eriksson et al., 2019, p. 65). Machines both automate the creation of playlists, making their production more efficient, and improve-like an "exoskeleton"-the skills of human curators, making them faster in their choices and speeding up production times. Pelly (2017), after a conversation with a playlist creator for Spotify, recounted that "these human curators are responding to data to such an extent that they're practically just facilitating the machine process." On the other side, humans intervene on automatic playlists to make their output less predictable and constantly improve their code.

Music curation in the age of platformization is determined by these mutually shaping logics. Instead of contrasting editorial and algorithmic logics, we should thus frame these logics as stacked and entangled, both shaping the outputs of platforms. These are always present together, but with different weights. Each platform articulates these logics by giving them a different relevance. In some Spotify playlists, the algorithmic logic weighs more, while in other playlists, editorial logics are more relevant.

Drawing from the expression used by Daboh, above (in Ramirez, 2017), we define this combined logic as an "algotorial" one. During our field research, we heard this term many times: it also appeared in an article on music curators published by NPR News: "Spotify's playlist content is determined by a staff of editorial tastemakers, in combination with a suite of proprietary machine-learning algorithms, an approach to song selection that Spotify execs describe with the gruesome neologism "algo-torial" (Witt, 2018). We understood this term as an "experience near" concept (Geertz, 1974), a word spontaneously used by our informants to describe their activity, and we immediately found it relevant to our inquiry. This algo-torial logic is clearly shown in the description of the work performed by the Spotify curators that oversee the compilation of the playlist "New Music Friday." As one informant told us,

They (curators, nda) are very important in week one. After week one the algorithms kick in to tell us what we need to do. Spotify is very dependent upon editorial for week one and then the 
algorithms take the lead in week two. (Informant B, digital music promoter)

When Informant B pronounced the words "week one is editorial, week two is algorithmic," we thought this was key to understand how the gatekeepers of the "platformed" cultural industries operate. ${ }^{5}$ This entanglement between editorial and algorithmic logics can be witnessed in the position a song is given within a playlist. In the first week, it appears on a certain playlist; the position of a song depends on the curator's choices. In the meantime, the algorithm evaluates the song's performance based on a number of parameters, such as the number of plays, the number of skips received, the amount of plays completely finished, the time spent listening, the amount of users that included the song among their favorites, and the "passive or intentional modality of listening" (Informant P, Apple). As a curator at Google Play Music told us, the most relevant data to decide where to position a song into a playlist are engagement, impressions, listening duration, and skip rates. He told us that he received specific internal training to be able to "make sense of the data."

Google Play curators periodically take part in strategic planning meetings, where key performance indicators (KPIs) are established. In these meetings, the curators are instructed to give maximum value to engagement and to constantly keep an eye on it:

I have at my disposal a dashboard for analytics and I monitor, day by day, the overall performance of my playlists. Every week I export data to analyze the behavior of each single playlist, then I implement adjustments for the non-performing ones. (Informant N, Google Play)

The position of a song on a playlist in turn seems to play a major role in determining its visibility (and, consequently, that of the artist). Numerous curators have confirmed this aspect: "Position matters, completely. We are obsessive about it," a Spotify curator said to Allen (2017). "When creating playlists, I probably spend the most time on the order. The data might tell you that people are skipping or stopping listening, but an algorithm wouldn't necessarily know why, or how to fix it" (Dredge, 2016). Importantly, the position of a song within a playlist is not a fixed one, as it used to be in records' tracklists. Positioning within a playlist varies over time, according to the mixed action of algorithmic logics based on users' feedbacks and editorial logics based on curators' skills. In this sense, the position of a song is "contingent," as intended by Nieborg and Poell (2018): "increasingly modular in design and continuously reworked and repackaged, informed by datafied user feedback" (p. 1). These two logics act in real time on the generation and the curation of the playlist: the playlists, a week after the release, no longer have the same shape.

The awareness of the mutual shaping of the two logics can also be found in the words of another informant: "I think really, these two things [human and algorithmic curation, Author's note] are mutually dependent on each other increasingly, because you need the algorithm to do the heavy lifting. It's a symbiosis, right?" (Informant I, ex-Universal).

\section{The Algo-torial Power of Platform Gatekeepers: New Regimes of Visibility}

Tuchman (1978), in a way similar to the theory of agenda setting (McCombs \& Shaw, 1972), argued that "News making imposes a frame for defining and constructing social reality" (1978, p. 180). We suggest the same idea applies to digital platforms dealing with cultural products. The ability of digital platforms like Facebook and Twitter to set the agenda has already been shown by Wohn and Bowe (2014, 2016), who described how platforms like Twitter and Facebook contribute to the social construction of reality and act as "micro-agenda setters." Music streaming platforms seem to be able to similarly shape the global agendas of music consumption: just as legacy media "may not be successful much of the time in telling people what to think, but it is stunningly successful in telling its readers what to think about" (Cohen, 1963, p. 13), music streaming platforms may not be directly successful in telling people what music to like, but nevertheless can be stunningly successful in telling its users what is worth listening to. When a music curator and/or an algorithm places a song at the top of a Spotify playlist like "New Music Friday" or "Rap Caviar" and assigns less visible positions to others, this creates not just a numerical but also a cultural hierarchy of importance of those songs.

The platformization of music curation imposes therefore new "regimes of visibility" (Bucher, 2012) and intensifies what Bucher (2018) calls the "threat of invisibility": algorithms and curators decide and discipline the visibility of an artist within the platform. ${ }^{6}$ This agenda-setting role is openly recognized by the insiders of the music sector, such as Larry Miller, who heads the music business program at New York University's Steinhardt School and who claimed that the,

Most important gatekeeper in the music business right now is Tuma Basa, the global head of hip-hop at Spotify (currently at YouTube, Authors' note). With around 8.3 million followers, the playlist sets the agenda for hip-hop the way New York radio station HOT 97 once did. (Shah, 2017)

Platform gatekeepers decide, filter, and select what to expose to the public and what cultural item to direct their attention to.

Besides, the algo-torial power of music streaming platforms is not only able to directly influence the agenda of music listeners, it could also indirectly affect it by inspiring and shaping the listening agenda of music radio programmers. During our brief period of participant observation at both Rai Radio 2 and BBC Radio 6, our informants confirmed that their curation choices are still guided in part by 
personal taste and editorial decisions, but are increasingly influenced also by data coming from music streaming platforms: not only YouTube or Soundcloud, but also Spotify, Apple Music, and Shazam. Shazam, our informants revealed, has a particularly important positioning in the music industry as it is believed to be able to predict the success of new talents up to 11 months before they appear on mainstream outlets, such as BBC playlists. As our informants at Shazam confirm,

BBC Radio 1 presenters use Shazam data for some of the artists and songs they put on the radio, so Shazam has definitely contributed to the radio plays of some artists and especially unsigned artists. Apple, Deezer, Spotify curators too check our trends. (Informants $\mathrm{C}$ and D)

Shazam's data are also being used by music festival organizers, to aid their gatekeeping activity (deciding the next lineup), and by music labels, to discover new talent. Informant B confirmed that curators at traditional media outlets base their editorial choices on data from music streaming platforms:

Today, if you walk in to a radio station, they will literally ask you, what're your Spotify numbers, is the song up on the services it's streaming, how many followers, show me your social media. The music industry's always been data driven. It's just that there's new data and more orbits. (Informant B, digital music promoter)

In other words, the power of music streaming platforms to set the listening agenda of both music radio programmers and music consumers is not mainly computational (Tufekci, 2015) or algorithmic (Lash, 2007), but eminently cultural, as a result of the blending of the editorial with the algorithmic logics and the power music curators yield in this encounter. The algo-torial agency of these platforms is located within a field (the music industry) crossed by different power relations, where different actors "struggle" to gain temporary vantage points. The output of this "struggle" is a playlist in which these power relations are finally "coded" in. The power balance between the ability of platforms to structure consumption and the individual agency of music listeners changes from platform to platform and evolves over time. Each platform, according to its productive routines, vision, and type of audience, wields a different type of power that can be more or less tilted toward editorial or algorithmic logics, as we showed in the previous section.

The output of this power-the playlist-is a contingent commodity (Nieborg \& Poell, 2018), always open to revision. Likewise, the dominion exerted by music streaming platforms on the listening agenda of its users is also contingent: the asymmetry of power between platforms and users could perhaps be best understood as an unstable balance of power between the platform and its users. Yet, it must be noted that while commercial music streaming platforms are extending their hegemonic position in the field of music consumption, there are already signs of resistance to this dependence upon platforms, as Kitchin (2017, p. 19) predicted. Music labels, musicians, promoters, and listeners, as showed by Ingham (2018a), engage in practices aimed at "gaming" the algo-torial logic that could represent a fruitful issue for future research. For example, one of our informants (a music promoter) revealed that he ran a competition among the fans of one of his artists and asked them to guess how many times the artist mentioned a certain word in his new song just released on Spotify. Fans had to play that song many times to find the right answer and win the prize: "they listened to it multiple times in a row until they spot the word. This triggered the algorithms to put it in people's algorithmic playlist the following week, which drove another wave of streams." These practices, when intentionally aimed at subverting the output of the algorithms, or politically questioning the business model coded in these algorithms, could be labeled as "counter-hegemonic" in the sense intended by Mouffe (2011): "every hegemonic order is susceptible of being challenged by counter-hegemonic practices, that is, practices that will attempt to disarticulate the existing order so as to install other forms of hegemony" (p. 18).

\section{Conclusion}

This article has provided an in-depth account of the logics of music curation inside music streaming platforms. It has evidenced how curators of these platforms represent a new élite in the wider family of music gatekeepers. It has shown how platformed music curation is a "stacked" combination of editorial and algorithmic logics. On the basis of this evidence, we have argued that platform gatekeepers exert a kind of "algo-torial power" that may be able, as a primary consequence, to set the "listening agendas" of global music consumers. While the entanglement between data and "gut instinct" is nothing new in the music industry, we show that the specific workings of this entanglement are innovative and have broader implications for the platformization of culture. The nexus between humans and machines is a central issue for further research in the platformization of culture. We think this is better understood if framed not as a dualistic opposition (machines vs humans) but as a complex relationship, in which machines automate some human skills while, at the same time, act as an increasingly influential aid for human decisions and extend productive capacities.

While the combination of human labor power and machinery is typical of industrial capitalism, even before the rise of online platforms (Eriksson et al., 2019, p. 65), what is new here is the relevance that automation processes fueled by data and organized by algorithms have acquired within platformed cultural industries. The decisions of platform gatekeepers are supported by an array of data and analytics previously unknown to traditional gatekeepers. Pelly (2017), after a conversation with a playlist creator for Spotify, recounted that "human curators are responding to data to 
such an extent that they're practically just facilitating the machine process." In the same fashion, one informant told us that "the culture of having faith in data is the first thing I learned here." While the power of traditional gatekeepers was mainly of an editorial nature, albeit data had some relevance in orienting their choices, the power of platform gatekepeers is an editorial power "augmented" and enhanced by algorithms and big data. Platform gatekeepers have more data, more tools to manage and to make sense of these data, and thus more power than their predecessors. Platformization of music curation then consists of a data-intense gatekeeping activity, based on different mixes of algo-torial logics, that produces new regimes of visibility. This makes the platform capitalistic model (Srnicek, 2017) potentially more efficient than industrial capitalism in transforming audience attention into data and data into commodities.

Studying the entanglement between human and nonhuman curatorial work at music streaming services is therefore not only useful to understand the evolution of the contemporary music industry but also to better frame the broader processes of platformization of cultural industries. This study suggests new research questions: how do other online platforms involved in cultural production articulate the nexus between algorithmic affordances and human agency? What kind of power emerges from this entanglement? And what impact on cultural production and society at large does the exercise of this power have?

\section{Acknowledgements}

This paper has been discussed with many peers and friends. We would like to thank Indrek Albrus for hosting one of the authors as a visiting researcher at MEDIT Center of Tallinn University, where the first version of this paper was mostly written. We would also like to thank all the scholars that took part in the seminar organized by David Nieborg and Thomas Poell at the University of Toronto for their passionate and fruitful comments, especially Robert Prey and Tarleton Gillespie. Finally, we would like to thank all the anonymous informants that made this research possible. Without them, no black-box-unpacking practice would have been possible.

\section{Declaration of Conflicting Interests}

The author(s) declared no potential conflicts of interest with respect to the research, authorship, and/or publication of this article.

\section{Funding}

The author(s) received no financial support for the research, authorship, and/or publication of this article.

\section{ORCID iD}

Tiziano Bonini (iD https://orcid.org/0000-0002-0636-0555

\section{Notes}

1. Playlists on Spotify and other music streaming services are not only generated by the platform staff but also by brands and end users. However, on Spotify, user-generated playlists are marginalized in favor of those produced by the platform' staff. Spotify's Twitter account only promotes its own playlists, and when a Premium user opens the Spotify's browser, only playlists produced by the platform are suggested (Eriksson et al., 2019; Pelly, 2017).

2. All conversations were recorded with the consent of the interviewees and lasted between 45 and $80 \mathrm{~min}$. Names of the interviewees have been fully anonymized to preserve confidentiality. We can provide the lists of the questions we made to the interviewees for further replication and extension of this research.

3. We also acknowledge that the curators we tried to investigate here represent only a relatively small portion of the nodes of the complex network of the gatekeepers, gatewatchers, and networked gatekeepers that structure the circulation of cultural products in the field of music industry.

4. For "personal taste-driven selection," the curator intended those tracks he selected according to his personal taste, knowledge, and 'gut'; for "editorially-driven selection," he intended those tracks he selected according to the genre of the playlist he was compiling: if he was working on a r'n'b playlist, he could not select a r'n'r song; for "algorithmically-driven selection," he intended those tracks he selected according to the tracks' suggestions coming from the proprietary software of the platform.

5. Relying on editorial curation at the very beginning of the playlist is also a technical solution to what developers of recommendation systems call "the cold start problem"- there is not enough data at the beginning for an algorithm to work, so the system requires some editorial curation initially. But our informant told us that the curators keep on monitoring the playlist in the following days and could edit playlists even in Week 2.

6. This regime of visibility imposed by the platforms is clearly recognized by the artists. We found evidences of musicians addressing music curators on Twitter and begging them to listen to their songs. This regime also influences what artists decide to write and music labels to produce. In fact, musicians have started to change the way they write songs, as one informant told us:

People are putting choruses at the beginning of songs now, more so than after a verse. Because the first five seconds, if the listeners hear a chorus, then they're more likely to carry on listening. The reason they're doing that is because then you'll get kept in playlists. So the music itself has been altered to complement the platform in which it's going to get listened to the most on. (Informant $\mathrm{H}$ )

\section{References}

Airoldi, M., Beraldo, D., \& Gandini, A. (2016). Follow the algorithm: An exploratory investigation of music on YouTube. Poetics, 57, 1-13.

Allen, C. (2017, July 11). I create Spotify playlists for a living. Buzzfeed News. Retrieved from https://www.youtube.com/ watch? $\mathrm{v}=\mathrm{Ji}$ _WfHxatoQ

Altheide, D. (1976). Creating reality: How television news distorts events. Beverly Hills, CA: SAGE. 
Arvidsson, A., \& Bonini, T. (2015). Valuing audience passions: From Smythe to Tarde. European Journal of Cultural Studies, $18,158-173$.

Barna, E. (2017). "The perfect guide in a crowded musical landscape": Online music platforms and curatorship. First Monday, 22(4). Retrieved from http://firstmonday.org/ojs/index.php/fm/ article/view/6914

Barzilai-Nahon, K. (2008). Toward a theory of network gatekeeping: A framework for exploring information control. Journal of the American Society for Information Science and Technology, $59,1493-1512$.

Beer, D. (2017). The social power of algorithms. Information, Communication \& Society, 20, 1-14. doi:10.1080/13691 18X.2016.1216147

Bucher, T. (2012). Want to be on the top? Algorithmic power and the threat of invisibility on Facebook. New Media \& Society, $14,1164-1180$.

Bucher, T. (2016). Neither black nor box: Ways of knowing algorithms. In S. Kubitschko \& A. Kaun (Eds.), Innovative methods in media and communication research (pp. 81-98). London, England: Palgrave Macmillan.

Bucher, T. (2017). The algorithmic imaginary: Exploring the ordinary affects of Facebook algorithms. Information, Communication \& Society, 20, 30-44.

Bucher, T. (2018). If . . . then: Algorithmic power and politics. Oxford, UK: Oxford University Press.

Cohen, B. (1963). The press and foreign policy. New York, NY: Houghton Mifflin Harcourt.

Dredge, S. (2016, May 23). The new tastemakers: A day in the life of a music-streaming playlister. The Guardian. Retrieved from https:/www.theguardian.com/technology/2016/may/23/ music-streaming-services-playlister-sam-lee-deezer

Eriksson, M., Fleisher, R., Johansson, A., Snickars, P., \& Vonderau, P. (2019). Spotify teardown: Inside the black box of streaming music. Cambridge, MA: MIT Press.

Fiegerman, S. (2014, May 14). The slow decline of peer-to-peer file sharing. Mashable. Retrieved from https://mashable.com/2014/05/14/ file-sharing-decline/?europe=true\#QT40ILZoAaql

Fleischer, R. (2017). If the song has no price, is it still a commodity? Culture Unbound: Journal of Current Cultural Research, 9, 146-162.

Fleischer, R., \& Snickars, P. (2017). Discovering Spotify: A thematic introduction. Culture Unbound: Journal of Current Cultural Research, 9, 130-145.

Gans, H. J. (1979). Deciding what's news: A study of CBS evening news, NBC nightly news, newsweek, and time. New York, NY: Random House.

Geertz, C. (1974). "From the native's point of view": On the nature of anthropological understanding. Bulletin of the American Academy of Arts and Sciences, 28, 26-45.

Gillespie, T. (2014). The relevance of algorithms. In T. Gillespie, P. Boczkowski, \& K. Foot (Eds.), Media technologies: Essays on communication, materiality, and society (pp. 167-193). Cambridge, MA: MIT Press.

Gillespie, T. (2018). Custodians of the internet. New Haven, CT: Yale University Press.

Gitlin, T. (1980). The whole world is watching: Mass media in the making \& unmaking of the new left. Berkeley: University of California Press.
Glaser, B. G., \& Strauss, A. L. (1967). The discovery of grounded theory: Strategies for qualitative research. Chicago, IL: Aldine Press.

Hallinan, B., \& Striphas, T. (2016). Recommended for you: The Netflix Prize and the production of algorithmic culture. New media \& society, 18(1), 117-137.

Hannerz, U. (2003). Being there . . . and there . . . and there! Reflections on multi-site ethnography. Ethnography, 4, 201216

Hesmondhalgh, D., \& Meier, L. M. (2018). What the digitalisation of music tells us about capitalism, culture and the power of the information technology sector. Information, Communication \& Society, 21, 1555-1570.

Hogan, M. (2015, July 16). Up next: How playlists are curating the future of music. Pitchfork. Retrieved from https://pitchfork. com/features/article/9686-up-next-how-playlists-are-curatingthe-future-of-music/

Ingham, T. (2018a, February 20). The great Spotify scam: Did a Bulgarian playlister swindle their way to a fortune on streaming service? Music Business Worldwide. Retrieved from https:// www.musicbusinessworldwide.com/great-big-spotify-scambulgarian-playlister-swindle-way-fortune-streaming-service/

Ingham, T. (2018b, March 25). The odds of an artist becoming a "top tier" earner on Spotify today? Less than 1\%. Music Business Worldwide. Retrieved from https://www.musicbusinessworldwide.com/the-odds-of-an-artist-becoming-a-top-tier-earneron-spotify-today-less-than-1/

Kitchin, R. (2017). Thinking critically about and researching algorithms. Information, Communication \& Society, 20, 14-29.

Lash, S. (2007). Power after hegemony: Cultural studies in mutation. Theory, Culture \& Society, 24(3), 55-78.

Lewin, K. (1947). Channels of group life. Human Relations, 1, $143-153$.

Lucerne, A. (2017, January 24). Playlist pump: The Spotify pitching service. Upside Music. Retrieved from https://web.archive. org/web/20190307041929/http://www.upsidemusic.com.au/ blog/2017/1/24/playlist-pump-the-spotify-pitching-service

Marcus, G. E. (1995). Ethnography in/of the world system: The emergence of multi-sited ethnography. Annual Review of Anthropology, 24, 95-117.

McCombs, M. E., \& Shaw, D. L. (1972). The agenda-setting function of mass media. Public Opinion Quarterly, 36, 176-187.

McQuail, D. (1994). Mass communication theory: An introduction (3rd ed.). London, England: SAGE.

Meraz, S., \& Papacharissi, Z. (2013). Networked gatekeeping and networked framing on\# Egypt. The International Journal of Press/Politics, 18, 138-166.

Morris, J. W. (2015). Curation by code: Infomediaries and the data mining of taste. European Journal of Cultural Studies, 18, 446-463.

Mouffe, C. (2011). On the political. London, England: Routledge.

Mulligan, M. (2015). Awakening: The music industry in the digital age. London, England: MIDiA Research.

Napoli, P. M. (2014). Automated media: An institutional theory perspective on algorithmic media production and consumption. Communication Theory, 24, 340-360.

Nieborg, D. B., \& Poell, T. (2018). The platformization of cultural production: Theorizing the contingent cultural commodity. New Media \& Society, 20, 4275-4292. 
Pasquale, F. (2015). The black box society. Harvard: Harvard University Press.

Pelly, L. (2017, June 21). Not all Spotify playlists are created equal. Cash Music.com. Retrieved from https://watt.cashmusic.org/ writing/thesecretlivesofplaylists

Petre, C. (2015). The traffic factories: Metrics at Chartbeat, Gawker media, and the New York Times. New York, NY: Tow Foundation.

Popper, B. (2015, September 30). Tastemaker: How Spotify's discover weekly cracked human curation at internet scale. The Verge. Retrieved from https:/www.theverge.com/2015/9/30/9416579/ spotify-discover-weekly-online-music-curation-interview

Prey, R. (2017). Nothing personal: Algorithmic individuation on music streaming platforms. Media, Culture \& Society, 40, 1086-1100. doi:10.1177/0163443717745147

Prey, R. (2018, October). Platform pop. Paper presented at the Platformization of Cultural Production Workshop, Toronto, Ontario, Canada.

Ramirez, A. (2017, March 31). Algorithms in music: Is it the future? Vinyl List. Retrieved from https://web.archive.org/ web/20171229083655/http://vinyllist.com/

Schlesinger, P. (1978). Putting “reality” together. London, England: Methuen.

Schwartz-Shea, P., \& Yanow, D. (2013). Interpretive research design: Concepts and processes. London, England: Routledge.

Seaver, N. (2013, April). Knowing algorithms. Paper presented at Media in Transition 8, Cambridge, MA. Retrieved from http:// nickseaver.net/papers/seaverMiT8.pdf

Seaver, N. (2017). Algorithms as culture: Some tactics for the ethnography of algorithmic systems. Big Data \& Society, 4(2), 1-12. doi:10.1177/2053951717738104

Seaver, N. (2018). Captivating algorithms: Recommender systems as traps. Journal of Material Culture, 24, 421-436.

Shah, N. (2017, November 15). The music industry's new gatekeepers. The Wall Street Journal. Retrieved from https:// www.wsj.com/articles/the-music-industrys-new-gatekeepers-1510761601

Shoemaker, P. J., Vos, T., \& Reese, P. (2008). Journalists as gatekeepers. In K.W. Jorgensen \& T. Hanitzsch (Eds.), The handbook of journalism studies (pp. 73-87), New York, NY: Routledge.

Srnicek. (2017). Platform capitalism. London, England: Polity press.

Szczepanik P. (2013) On the ethnography of media production: An interview with georgina born. Illuminace, 25(3), 99-119.

Tiffany, K. (2017, November 13). Three nights following Spotify playlist editors around New York's live music scene. The Verge. Retrieved from https://www.theverge .com/2017/11/13/16617900/spotify-playlist-curation-nyc-liveshows-fresh-finds-indie-latin-new-music

Tuchman, G. (1978). Making news: A study in the construction of reality. New York, NY: Free Press.

Tufekci, Z. (2015). Algorithmic harms beyond Facebook and Google: Emergent challenges of computational agency. Journal on Telecommunication and High Technology Law, 13, 203-218.

Ugwu, R. (2016, July 13). Inside the playlist factory. Buzzfeed. Retrieved from https:/www.buzzfeed.com/reggieugwu/theunsung-heroes-of-the-music-streaming-boom?utm_term=. rad4rya5k\#.fjJxDR256

van Dijck, J., Poell, T., \& de Waal, M. (2018). The platform society: Public values in a connective world. Oxford, UK: Oxford University Press.

White, D. M. (1950). The gatekeeper: A case study in the selection of news. Journalism Quarterly, 27, 383-390.

Witt, S. (2018, April 4). Spotify is, for now, the world's most valuable music company. NPR. Retrieved from https:/www.npr. org/sections/therecord/2018/04/04/599385111/spotify-is-fornow-the-worlds-most-valuable-music-company

Wohn, D. Y., \& Bowe, B. J. (2014). How social media facilitates social construction of reality. In Proceedings of the companion publication of the 17th ACM conference on computer supported cooperative work \& social computing (pp. 261264), Baltimore, MD: Association for Computing Machinery Press.

Wohn, D. Y., \& Bowe, B. J. (2016). Micro agenda setters: The effect of social media on young adults' exposure to and attitude toward news. Social Media + Society, 2(1), 1-12. doi: $10.1177 / 2056305115626750$

\section{Author Biographies}

Tiziano Bonini ( $\mathrm{PhD}$, University of Siena) is associate professor in Sociology of Media and Culture at the Department of Social, Political and Cognitive Sciences at the University of Siena. His research interests include radio studies, political economy of the media, media production studies, and digital cultures.

Alessandro Gandini (PhD, University of Milan) is a senior researcher in Digital Sociology at the Department of Social and Political Sciences at the University of Milan, Italy. Previously, he was lecturer in Digital Media Management and Innovation in the Department of Digital Humanities, King's College, London. His research focuses on the encounter between digital technologies and society and particularly on the evolution of work in the digital age. His latest book, entitled Zeitgeist Nostalgia: On populism, work and the 'good life', is forthcoming (2020) with Zero Books. 